\title{
Automated segmentation and classification technique for brain stroke
}

\author{
N. S. M. Noor', N. M. Saad ${ }^{2}$, A. R. Abdullah ${ }^{3}$, N. M. Ali ${ }^{4}$ \\ ${ }^{1,2}$ Fakulti Kejuruteraan Elektronik dan Kejuruteraan Komputer, Universiti Teknikal Malaysia Melaka, Malaysia \\ ${ }^{3,4}$ Fakulti Kejuruteraan Elektrik, Universiti Teknikal Malaysia Melaka, Malaysia
}

\section{Article Info \\ Article history: \\ Received Sep 2, 2018 \\ Revised Dec 16, 2018 \\ Accepted Jan 26, 2019}

\section{Keywords:}

Brain imaging

Classification

Diffusion-weighted imaging

(DWI)

Segmentation

Stroke

\begin{abstract}
Difussion-Weighted Imaging (DWI) plays an important role in the diagnosis of brain stroke by providing detailed information regarding the soft tissue contrast in the brain organ. Conventionally, the differential diagnosis of brain stroke lesions is performed manually by professional neuroradiologists during a highly subjective and time- consuming process. This study proposes a segmentation and classification technique to detect brain stroke lesions based on diffusion-weighted imaging (DWI). The type of stroke lesions consists of acute ischemic, sub-acute ischemic, chronic ischemic and acute hemorrhage. For segmentation, fuzzy c-Means (FCM) and active contour is proposed to segment the lesion's region. FCM is implemented with active contour to separate the cerebral spinal fluid (CSF) with the hypointense lesion. Pre-processing is applied to the DWI for image normalization, background removal and image enhancement. The algorithm performance has been evaluated using Jaccard Index, Dice Coefficient (DC) and both false positive rate (FPR) and false negative rate (FNR). The average results for the Jaccard index, DC, FPR and FNR are $0.55,0.68,0.23$ and 0.23 , respectively. First statistical order method is applied to the segmentation result to obtain the features for the classifier input. For classification technique, bagged tree classifier is proposed to classify the type of stroke. The accuracy results for the classification is $90.8 \%$. Based on the results, the proposed technique has potential to segment and classify brain stroke lesion from DWI images.
\end{abstract}

Copyright $(0) 2019$ Institute of Advanced Engineering and Science. All rights reserved.

\section{Corresponding Author:}

N. M. Saad,

Fakulti Kejuruteraan Elektronik dan Kejuruteraan Komputer, Universiti Teknikal Malaysia Melaka, Melaka, Malaysia.

Email: norhashimah@utem.edu.my

\section{INTRODUCTION}

Nowadays, medical imaging tool plays an important role in viewing the internal tissue of human brain. In brain stroke diagnosis, medical imaging such as MRI offers fast imaging tool with high resolution and nonionizing radiation [1]. The MRI imaging tool is able to detect $85 \%$ stroke survivor from ischemic stroke and $15-20 \%$ stroke survivor from hemorrhagic stroke [2]. DWI is one of the MRI modality that shows high sensitivity (88-100\%) and specificity (95-100\%) in detecting early ischemic stroke [3].

To fully utilize medical imaging, new algorithms and methods are urged in the medical imaging field for utilizing very large amounts of images wisely [4]. However, due to the large sample of images, the diagnosis process is time consuming and tiredness [5]. A computer aided diagnosis is needed for neuroradiologist to interpret the images more easily. Many researchers have studies machine learning technique in brain imaging [6]. Machine learning technique is believed to perform tasks and solve problems related to poor quality brain imaging samples by providing accurate representation and prior knowledge modeling. Thus, it plays an important role in brain imaging where it has become one of the most promising research areas in computer aided detection and diagnosis. 
Zhang Y. et al. [7] proposed kernel support vector machine to detect Alzheimer's disease (AD) and classify the disease between normal elder control (NC). Ramani R.G. et al. [8] proposed Naive Bayes, Support Vector Machine and random tree to analyze the machine learning technique in classifying normal and abnormal brain image. Mudali D. et al. [9] proposed decision tree method to interpret the diagnosis of neurodegenerative brain diseases. Meet Oza et al. [10] proposed random forest classifier to classify brain tumor into benign or malign cases. Fratello M. et al. [11] proposed random forest to classify two type of disease from individual lateral sclerosis (ALS) patients, Parkinson's disease (PD) patients and healthy control (HC) subjects.

In this paper, segmentation and classification technique for brain stroke using DWI image is proposed. The purpose of this study is to develop an automatic stroke segmentation and classification using DWI images. The proposed segmentation analysis is based on the fuzzy c-Means (FCM) and active contour technique. Active contour is integrated in the analysis framework to separate the cerebral spinal fluid (CSF) with the hypointense lesion and increase the segmentation accuracy. The performance of the segmentation techniques is evaluated based on Jaccardindex, Dice Coefficient (DC), false positive rate (FPR) and false negative rate (FNR). After the segmentation result is obtain, the result image is extracted using first order statistical method. These features are used for the bagged tree classifier input. The performance of the classifier technique is evaluated based on the accuracy (ACC).

This paper contains five section. Section 2 discussed the flow process of the proposed methodology in detail. Section 3 proposed the brain stroke analysis technique used to segment and classify the brain stroke lesion. Section 4 discussed the result from the segmentation result and Section 5 conclude the work presented.

\section{RESEARCH METHOD}

\subsection{Proposed analysis framework}

The flow process of this study starts with image preprocessing where image normalization, image enhancement and background removal are applied. After that, FCM is applied for image segmentation stage in order to extract the ROI of the stroke lesion. Then, the active contour is used to separate the CSF with the hypointense stroke lesion region. Hyperintense is referred to bright lesion such as acute ischemic stroke, acute hemorrhage stroke and sub-acute ischemic stroke lesion where hypointense is referred to dark lesion such as chronic ischemic stroke lesion. The segmentation result is extracted using first order statistical method for the input classifier. After the features are obtained, bagged tree classifier is used to classify the type of strokes lesion. Last but not least, the performance of the brain image is evaluated based on the Jaccard index, DC, FPR, FNR, ACC.

\subsection{Imaging parameter}

This paper focused on two brain stroke datasets from DWI images. Dataset 1 contains 61 samples from the General Hospital of Kuala Lumpur. Dataset 2 contains 69 samples from the online database of Ischemic Stroke Lesion Segmentation (ISLES). The dataset only focused on four types of brain stroke lesion which are acute ischemic stroke, chronic ischemic stroke, acute hemorrhage stroke and sub-acute ischemic stroke. The acute ischemic stroke, chronic ischemic stroke and acute hemorrhage stroke images were gained from the General Hospital of Kuala Lumpur (HKL). The sub-acute ischemic stroke images were gained from the public online Ischemic Stroke Lesion Segmentation (ISLES) challenge 2015 [12].

Table 1. The Brain Stroke Datasets

\begin{tabular}{lcccc}
\hline Stroke Datasets & Acute Ischemic & $\begin{array}{c}\text { Dataset 1 (clinical) } \\
\text { Chronic Ischemic }\end{array}$ & Acute Hemorrhage & $\begin{array}{l}\text { Dataset 2 (ISLES) [12] } \\
\text { Sub-acute Ischemic }\end{array}$ \\
\hline Number of Samples & 20 & 33 & 8 & 69 \\
\hline
\end{tabular}

\subsection{Image pre-processing}

The pre-processing stage is the stage where the images need to undergo pre-processing stages to acquire better segmentation [13]-[15]. Three algorithms were applied to the DWI image which is image normalization, background removal and image enhancement [16]. These images are converted into the desired form in which the intensity is adjusted and the noise is removed.

\subsection{Fuzzy c-means}

FCM is a clustering method where it divides a group of pixels into homogenous cluster and assigns the pixels according to their category. It allows pixels' points to be assigned to multiple clusters and each 
pixel point has a degree of membership in a cluster to which it belongs. In this segmentation technique, the pixel point is select base on the center of three cluster which are lower, middle and higher cluster. Each data point of the cluster should equal to one. It is based on minimization of the following objective function:

$$
J_{m}=\sum_{i=1}^{N} \sum_{j=1}^{C} u_{i j}^{m}\left\|x_{i}-c_{j}\right\|^{2}, 1 \leq m<0
$$

where $\mathrm{m}$ is any real number greater than $1, u_{i j}$ is the degree of membership of $x_{i}$ in the cluster $\mathrm{j}, x_{i}$ is the $i$ th of d-dimensional measured data, $c_{j}$ is the d-dimension center of the cluster, and $\|*\|$ is any norm expressing the similarity between any measured data and the center. Fuzzy partitioning is carried out through an iterative optimization of the objective function shown above, with the update of membership $u_{i j}$ and the cluster centers $c_{j}$ by:

$$
u_{i j}=\frac{1}{\sum_{k=1}^{c}\left(\frac{\left\|x_{i}-c_{j}\right\|}{\left\|x_{i}-c_{j}\right\|}\right)^{\frac{2}{m-1}}} ; c_{j}=\frac{\sum_{i=1}^{N} u_{i j}^{m} \cdot x_{i}}{\sum_{i=1}^{N} u_{i j}^{m}}
$$

This iteration will stop when,

$$
\max _{i j}\left\{\left|u_{i j}^{(k+1)}\right|-u_{i j}^{(k)}\right\}<\varepsilon
$$

where $\varepsilon$ is a termination criterion between 0 and 1 , whereas $k$ are the iteration steps.

\subsection{Active contour}

In DWI chronic stroke lesion image, the cerebrospinal fluid (CSF) share the similar intensity level with the stroke lesion. Due to this matter, FCM method has failed to segment the lesion since the algorithm in the cluster cannot be differentiated. To improve this performance, the CSF area is removed by using the active contour method.

Active contour is a method that create boundaries in an image. It uses computer generated curves to detect and locate object. This method is often using in medical images to find the boundaries of an organ in the images. The image is classified into two part which are object region and background region. In this method the region is represented as the inside and outside regions of the zero-level set.

Level set framework take two signs positive and negative to divide image domain. The image domain is separated into two disjoint region $\Omega 1$ and $\Omega 2$. Local intensity clustering property means that the intensities in the neighborhood $0_{y}$ can be divided into $\mathrm{N}$ clusters, with center $C_{i}, i=1,2, \ldots, N$. It can be written as:

$$
F_{y}=\sum_{i=1}^{N} \int_{0 y}\left|I(x)-c_{i}\right|^{2} m_{i}(x) d x
$$

Where $C_{i}$ the cluster in the center of the i-th cluster is, $m_{i}$ is the membership function of the region, i.e. $m_{i}(x)=1$ for $x \in \Omega_{i}$ and $m_{i}(x)=0$ for $x \notin \Omega_{i}$. Or the corresponding equation can be witten as:

$$
E_{\text {active contour }}=\int_{0}^{s_{m}}\left[E_{\text {int }}(v(s))+E_{\text {ext }}(v(s))\right] d s
$$

Where the position of active contour is describe parametrically by $\mathrm{v}(\mathrm{s})=(\mathrm{x}(\mathrm{s}), \mathrm{y}(\mathrm{s}))$, Eint represents internal potential energy of the contour, $E_{\text {ext }}$ is the energy that models external constraints impose onto the contour shape. $G(y-x)$ is the Gaussian kernel applied as window function showed.

$$
G(d)=\left\{\begin{array}{cc}
{ }_{a}^{1} e-\frac{|d|^{2}}{2 \sigma^{2}} & \text { for }|d| \leq \rho \\
0 & \text { otherwise }
\end{array}\right.
$$


Where ais a constant, $d$ is a distance between $\mathrm{x}$ and y points. $\sigma$ is a standard deviation or scale parameter of Gaussian function, and $\rho$ is a radius of neighboring pixels. The radius of the neighborhood should be choose appropriately based on the degree of intensity in-homogeneity.

The next step is contour construction to define initial shape around the object that will serve as an initialization set up. Last but not least, the greedy method is applied to simplify the implementation of the minimization of energy without having to perform an optimization algorithm method like gradient descent [17]-[21]. The function of this method is by finding for each point of the contour the closest local energy minimizing neighbor will converge to the overall global minimum of the contour. The method using 2 equations which are:

$$
E_{\text {int }}=\frac{\left(\alpha(s)\left|V_{s}(S)\right|^{2}+\beta(s)\left|V_{s}(S)\right|^{2}\right)}{2}
$$

Where $\alpha(s)$ represent adjusts the elasticity of the active contour and $\beta(s)$ is adjusts the stiffness of the active contour.

$$
E_{\text {ext }}=-\delta \int_{A}^{B}\left\|\nabla\left(G_{n} * I\right)\right\|^{2}(\gamma(s)) d s
$$

Where $\delta$ is a real weighting value which for obvious reason would be positive, $G n$ is a Gaussian weighted kernel of dimension $\mathrm{n}$, I represent the input image, $\nabla$ is the spatial gradient function and $\gamma(s)$ is the contour function.

\subsection{First order statistical method}

First order statistical order method is applied at the ROI to obtain several features. Since this ROI depends on the signal of intensity, the ROI is classified into two parts of the image which are hyperintense and hypointense lesion. Mean, median and mode are used to separate the image between hyperintense and hypointense lesion. For hyperintense lesion, standard deviation is assigned while in hypointense lesion mean of region boundary is assigned. The standard deviation and mean of boundary are used to differentiate each characteristic of stroke lesion.

The calculation for the first order method is shows as below:

$$
\begin{aligned}
& \mu=\frac{1}{N} \sum_{i=1}^{N-1} P(i) \\
& \text { Median }=\frac{1}{2}(n+1)^{\text {th }} \text { value } \\
& \sigma=\sqrt{\frac{1}{N} \sum_{i=0}^{N-1}(P(i)-\mu)^{2}} \\
& \text { Pearson mode skewness }=\frac{\mid \text { mode }-\mu \mid}{\sigma} \\
& \text { Compactness }=\frac{(\text { Length }(\text { boundary }))^{2}}{\text { Area }}
\end{aligned}
$$

Where $P(i)$ is the probability intensity level of the ROI, $N$ be the total number of gray levels in the entire ROI.

\subsection{Bagged tree classifier}

Bagged tree classifier is a method that perform a collection of data by analyze the whole data than individually. This classifier builds and trains a variety of decision trees and produces classes from the mode of individual tree class. It applies general techniques by resampling data from the previous learning. Assume that For $b=1, \ldots, B$; where $B$ is the bagging classifier ( $B$ times) that repeat the train set of $D=d_{1}, \ldots, d_{n}$ with 
the response $R=r_{1}, \ldots, r_{n}$. Each individual tree class will observe the data from the previous data of tree class and make their own decision. The decision that make is then is selected and being compare with the other tree classes by average the data. For unseen data $\left(x^{\prime}\right)$, the data is select by using the mod of data in the tree class.

$$
\hat{f}=\frac{1}{B} \sum_{b=1}^{B} f_{b}\left(x^{\prime}\right)
$$

In this experiment, the bagged tree classifiers train 129 data for the number of branch node splits per tree. Figure 1 shows the bagged tree diagram.

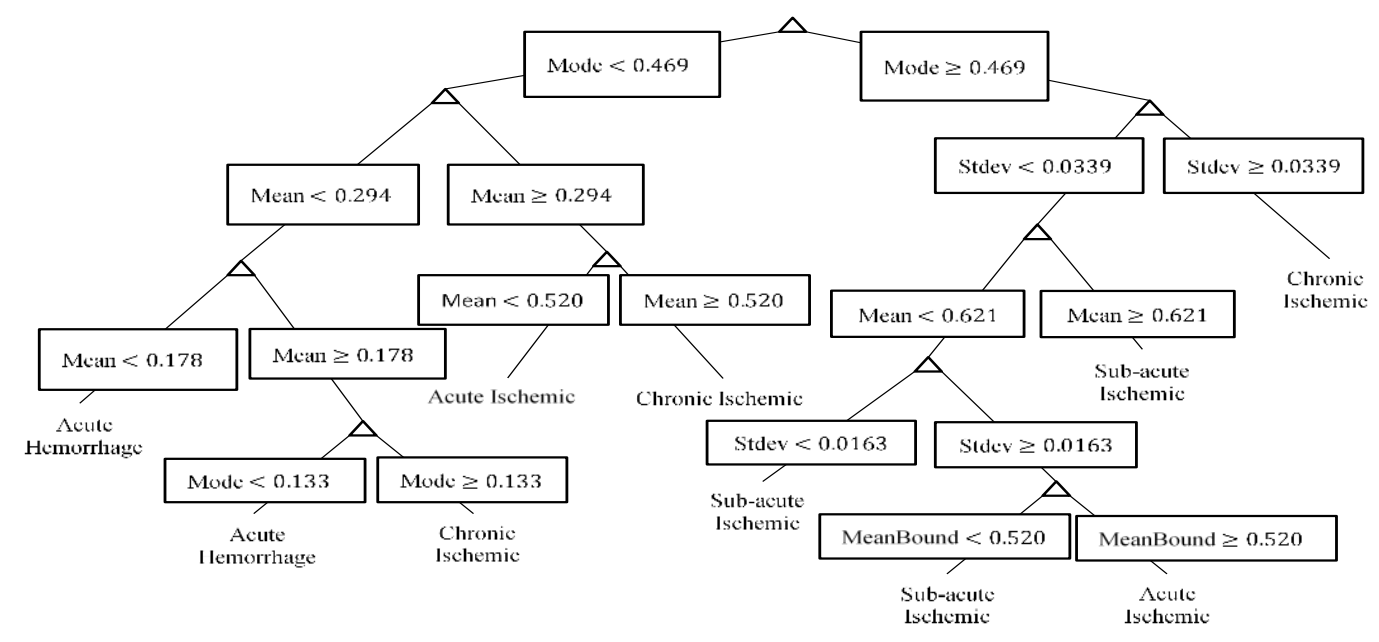

Figure 1. Bagged tree classifier diagram

\subsection{Performance evaluation}

The performance evaluationis calculated from the segmentation results with the neuroradiologist manual reference. Jaccard index, Dice coefficient (DC), false positive rate (FPR) and false negative rate (FNR) are used as the performance metrics [16]. From this calculation, the DWI segmentation result can be fully segment.

The performance verification for classification take part in the statistical calculation and shown in confusion matrix attributes using MATLAB APPS. Number of observations, true positive rate (TPR), false negative rate (FNR), positive predictive values (PPV) and false discovery rate (FDR) is used to identify the performance of the classifier. Below is the statistical calculation for performance verification.

$$
\begin{aligned}
& T P R=\frac{T P}{P} \\
& T N R=1-T P R
\end{aligned}
$$

Where true positive (TP) is the number of samples are correctly classified within their type of stroke in the positive sample (P). Positive predictive value (PPV) as shown in Equation (17) is calculated as the probability that correctly classify the type of stroke. The false positive (FP) is misclassified of stroke.

$$
\mathrm{PPV}=\frac{T P}{T P+F P}
$$

Lastly the performance of the classifier will be verified using $A_{c c}$ to as shown in Equation (18).

$$
A_{c c}=\frac{T P+T N}{P+N}
$$


Receiver operating characteristic (ROC) is used to indicate TPR and FPR for each type of stroke. From the year 1940, ROC graph is useful for interpreting medical test results. The recipient operating feature is the metric used to check the quality of the classification. For each type of stroke, ROC uses the threshold value along the interval 0 and 1 to the output. For each threshold, two values are calculated based on the TPR and the FPR formula.

$$
F P R=\frac{F P}{F P+T N}
$$

Where false positive $(F P)$ is the number of samples are misclassified within their type of stroke in the positive sample. True negative $(T N)$ is the number of samples are misclassified. In this ROC curve, TPR is a set of outputs greater or equal to the threshold, divided by a target value and $F P R$ is a set of outputs less than the threshold, divided by the zero-target value. The AUC is a result of the overall quality of the stroke. Larger AUC indicates better performance for the classifier.

$$
A U C=P\left(X_{1}>X_{0}\right)
$$

Where $X_{1}$ is the probability of score for the type of stroke that is classify into its class and $X_{0}$ is vice versa to $X_{1}$.

\section{RESULTS AND ANALYSIS}

\subsection{FCM and active contour segmentation technique}

The segmentation results of the original image from stroke lesion are showed in Figure 2. From the segmentation results, FCM can accurately segment the hyperintense lesion. The hyperintense lesion of DWI images shows high accuracy result compare to the hypointense lesion. Even though the result shows by the FCM toward hypointense lesion is low accuracy, the active contour technique can be used to separate the hypointense lesion with the CSF

Table 2 shows the performance analysis and evaluation of the proposed FCM segmentation method for DWI images from dataset 1 and dataset 2. The performance of the algorithm is measured using the metrics such as Jaccard index, DC, FPR and FNR.

The result shows the proposed method offers very good segmentation result for sub-acute ischemic stroke according to high value of Jaccard index and DC with low value of FPR and FNR. The hypointense lesion is less accurate compare to hyperintense lesion due to the FCM technique in producing the ROI image. The segmentation also shows that the stroke lesion is over segmentation based on the result show in FPR. However, the segmentation proposed still can segment the ROI of brain stroke. The active contour has successfully separated the CSF area with the hypointense lesion.

Table 2. The Performance Metrics for DWI Stroke Datasets

\begin{tabular}{llcccc}
\hline \multirow{2}{*}{ Dataset } & \multirow{2}{*}{ Type of Stroke } & \multicolumn{3}{c}{ Performance Metrics } \\
& & Jaccard index & DC & FPR & FNR \\
\hline \multirow{3}{*}{ Dataset 1} & Acute Ischemic & 0.53 & 0.66 & 0.09 & 0.37 \\
& Chronic Ischemic & 0.28 & 0.42 & 0.61 & $\mathbf{0 . 1 0}$ \\
& Acute Hemorrhage & 0.68 & 0.79 & 0.18 & 0.25 \\
Dataset 2 & Sub-Acute Ischemic & $\mathbf{0 . 7 2}$ & $\mathbf{0 . 8 3}$ & $\mathbf{0 . 0 5}$ & 0.23 \\
\hline
\end{tabular}




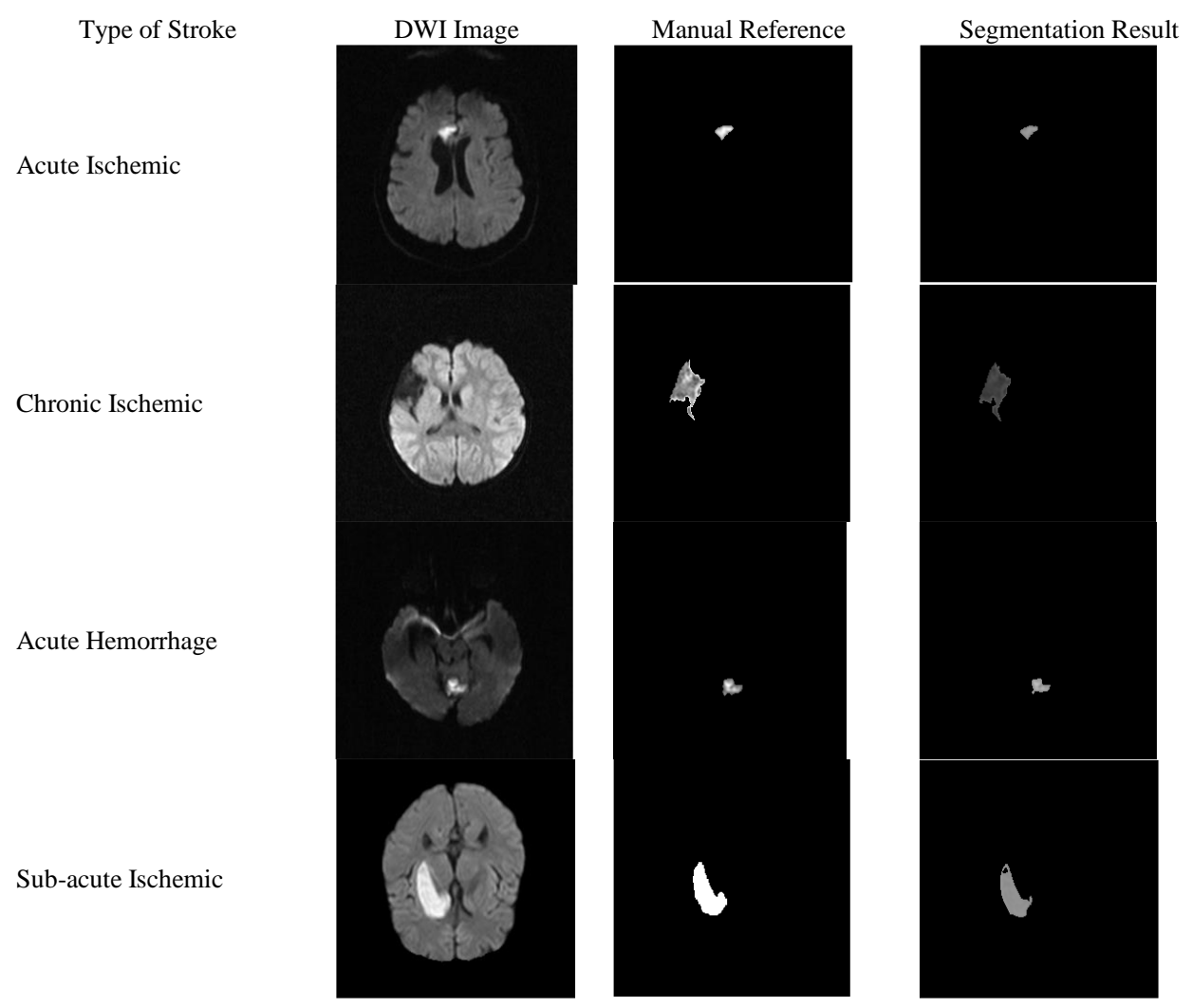

Figure 2. The segmentation results of the original image from DWI stroke lesion

\subsection{Bagged tree classifier}

The performance of the confusion matrices of the classification method is shown in Table 3 . Table 3 shows the number of observations of each type of stroke lesion. Only all chronic ischemic stroke samples are correctly classified into its group.5 sample of acute ischemic is misclassified into sub-acute ischemic lesion. For acute hemorrhage stroke only 3 sample areclassified correctly. 4 other sample are misclassified into subacute ischemic others into acute ischemic. For sub-acute ischemic stroke 67 sample is classified correctly within their groups, 1 sample is misclassified into acute ischemic and others 1 is misclassified into acute hemorrhage. The hyperintense lesion is mostly misclassified into their classes due to the range of features of each class that shares the same features with other class.

Table 3. The Performance Metric for Classification Method

\begin{tabular}{cccccc}
\hline & & Predicted Class & & \\
& Acute Hemorrhage & Acute Ischemic & Chronic Ischemic & Sub-acute Ischemic \\
\hline \multirow{4}{*}{ True Class } & Acute Hemorrhage & 3 & 1 & 4 & 5 \\
& $\begin{array}{c}\text { Acute Ischemic } \\
\text { Chronic Ischemic }\end{array}$ & & 15 & 33 & 67 \\
& Sub-acute Ischemic & 1 & 1 & & 67 \\
\hline
\end{tabular}

Table 4 shows the performance value of TPR, FNR, PPV and FDR of each type of stroke. Chronic ischemic stroke shows better performance base on higher value in TPR and PPV with lower value of FNR and FDR. The proposed classification method shows the performance result for acute ischemic, chronic ischemic and sub-acute ischemic is high compare to the acute hemorrhage. The TPR result for acute stroke show low value because the sample of acute hemorrhage refer to sub-acute ischemic is higher. However, the PPV of acute hemorrhage is close to 1 since that the acute hemorrhage is correctly diagnosed.

Figure 3 shows the ROC plot for each type of stroke. From the ROC plot, chronic stroke shows $100 \%$ on the TPR axis showing the stroke is correctly classified into its class. The positive type of each type of stroke for acute ischemic, chronic ischemic and acute hemorrhage is $2 \%, 1 \%$ and $15 \%$ respectively. Based on the result, acute ischemic, chronic ischemic and acute hemorrhage shows good ROC results since the 
result of FPR is close to the threshold value 1. The results for acute ischemic, chronic ischemic, acute hemorrhage and sub-acute ischemic stroke for AUC are 94\%, 100\%,89\%and 97\%, respectively. Overall the accuracy of the classifier proposed is $90.8 \%$.

Table 4. Performance Result for Classification Method

\begin{tabular}{ccccc}
\hline & TPR & FNR & PPV & FDR \\
\hline Acute Ischemic & 0.75 & 0.25 & 0.88 & 0.12 \\
Chronic Ischemic & $\mathbf{1}$ & $\mathbf{0}$ & $\mathbf{1}$ & $\mathbf{0}$ \\
Acute Hemorrhage & 0.38 & 0.63 & 0.75 & 0.25 \\
Sub-acute Ischemic & 0.97 & 0.03 & 0.88 & 0.12 \\
\hline
\end{tabular}

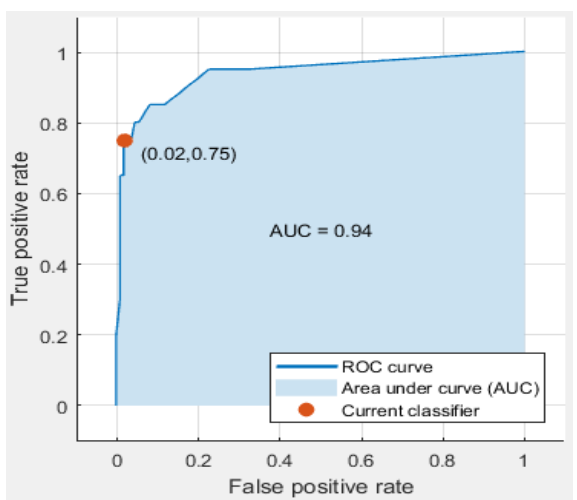

(a) ROC plot for acute ischemic

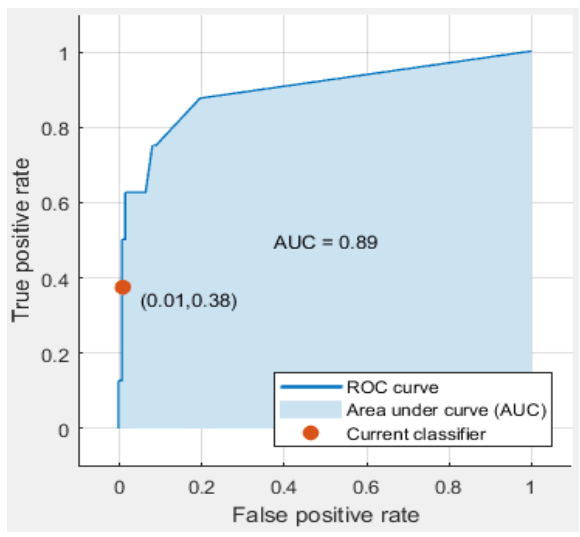

(c) ROC plot for acute hemorrhage

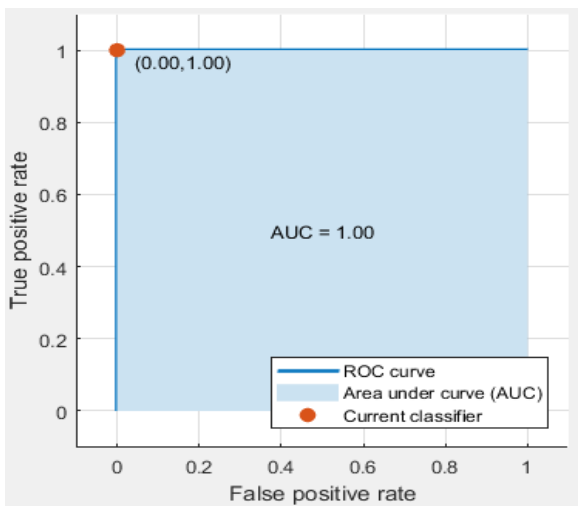

(b) ROC plot for chronic ischemic

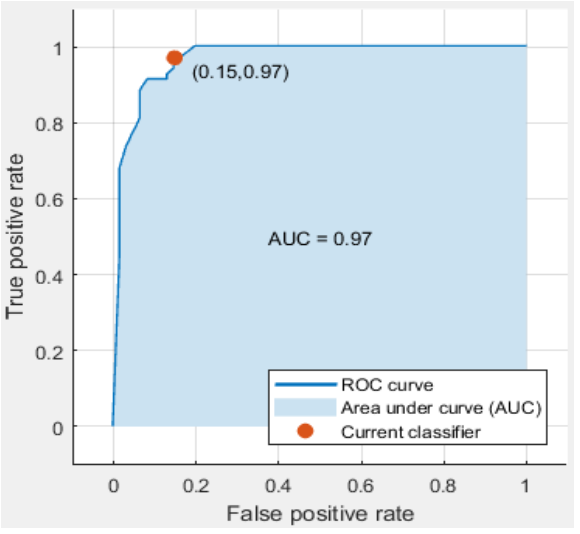

(d) ROC plot for sub-acute ischemic

Figure 3. ROC plot for each type of stroke

\section{CONCLUSION}

In this study, the FCM technique has been implemented with active contour to segment the stroke lesions in DWI images. The segmentation of active contour is implemented with FCM to separate the CSF area with the hypointense lesion. The segmentation results are compared with the manual reference to verify the accuracy. According to the above results, FCM provides good segmentation results in hyperintense lesions especially to sub-acute ischemic lesion according to the high value of Jaccard index and DC with low value of FPR and FNR presented in segment the brain stroke. Overall average values of Jaccard index, DC, FPR and FNR are $0.58,0.71,0.12$ and 0.31 respectively. Active contour technique shows that the brain stroke DWI image can segment the stroke lesion and separate the CSF wtith hypointense lesion well. Based on the features of the ROI segmentation result, each type of stroke is classified according to its class. Bagged tree classifier use ensemble method to predicted the type of each stroke lesion. All chronic stroke samples are correctly classified within it class. The accuracy show for this performance method is $90.8 \%$. 


\section{ACKNOWLEDGEMENTS}

The authors would like to thank to the Universiti Teknikal Malaysia Melaka (UTeM), Rehabilitation Engineering \& Assistive Technology (REAT) research group under Center for Robotics \& Industrial Automation (CeRIA), Advanced Digital Signal Processing (ADSP) Lab and Ministry of Higher Education (MOHE), Malaysia for sponsoring this work under project FRGS/1/TK04/FKE-CeRIA/F00334 and the use of the existing facilities to complete this project.

\section{REFERENCES}

[1] Gomes J, Wachsman AM,“Handbook of Clinical Nutrition and Stroke,"New York: Humana Press, Totowa, NJ,pp. 15-31,2013.

[2] Kanchana R, Menaka R.,"Computer Reinforced Analysis for Ischemic Stroke Recognition: A Review”,Indian Journal of Science and Technology,vol.8,no. 35, pp. 1-9,December 2015.

[3] Potente M, Gerhardt H, Carmeliet P.,"Basic and Therapeutic Aspects of Angiogenesis”,Cell, vol.146, no. 6, pp. 873-877, Sep 2011.

[4] Wernick MN, Yang Y, Brankov JG, Yourganov G, Strother SC.,"Machine Learning in Medical Imaging”,IEEE Signal Processing Magazine, vol. 27 no.4, pp.25-38, Jul 2010.

[5] Khademi A, Venetsanopoulos A, Moody AR, "Robust White Matter Lesion Segmentation in FLAIR MRI",IEEE Transactions on Biomedical Engineering., vol.59, no. 3, pp. 860-71, Mar 2012.

[6] Suzuki K., "Pixel-based Machine Learning in Medical Imaging" Journal of Biomedical Imaging, vol 1, Jan 2012.

[7] Zhang Y, Dong Z, Phillips P, Wang S, Ji G, Yang J, Yuan TF, "Detection of Subjects and Brain Regions Related to Alzheimer's Disease Using 3D MRI Scans Based on Eigenbrain and Machine Learning",Frontiers in Computational Neuroscience, vol. 2, Jun 2015.

[8] Ramani RG, Sivaselvi K., “Classification of Pathological Magnetic Resonance Images of Brain Using Data Mining Techniques",InRecent Trends and Challenges in Computational Models (ICRTCCM), 2017 Second International Conference, 2017,pp. 77-82, IEEE.

[9] Mudali D, Teune LK, Renken RJ, Leenders KL, Roerdink JB.,"Classification of Parkinsonian Syndromes from FDG-PET Brain Data Using Decision Trees with SSM/PCA Features”, Computational and Mathematical Methods in Medicine, 2015.

[10] Oza M, Kapdi R, Student B.,"Brain Tumor Disease Identification Using Random Forest Classifiers”, Brain, vol. 7 no. 1 , Sep 2015.

[11] Fratello M, Caiazzo G, Trojsi F, Russo A, Tedeschi G, Tagliaferri R, Esposito F.,"Multi-View Ensemble Classification of Brain Connectivity Images for Neurodegeneration Type Discrimination", Neuroinformatics, vol. 15, no. 2, pp.199-213, April 2017.

[12] Maier O, Menze BH, von der Gablentz J, Häni L, Heinrich MP, Liebrand M, Winzeck S, Basit A, Bentley P, Chen L, Christiaens D, "ISLES 2015-A Public Evaluation Benchmark for Ischemic Stroke Lesion Segmentation from Multispectral MRI", Medical Image Analysis, vol. 1, no. 35, pp. 250-690, Jan 2017.

[13] Liu J, Li M, Wang J, Wu F, Liu T, Pan Y, “A Survey of MRI-based Brain Tumor Segmentation Methods”,Tsinghua Science and Technology, vol. 19, no. 6, pp.578-95, Dec 2014.

[14] Baraiya N, Modi H., "Comparative study of Different Methods for Brain Tumor Extraction from MRI Images Using Image Processing”, Indian Journal of Science and Technology, Vol. 9, no. 4, Jan 2016.

[15] Rahman NA, Saad NM, Abdullah AR, Wahab FA., "The Internet of Things Beverages Bottle Shape Defect Detection using Naïve Bayes Classifier," International Journal of Human and Technology Interaction (IJHaTI), vol. 2, no. 1, pp. 71-6, Apr 2018.

[16] Saad N.M, Noor NS, Abdullah AR, Muda S, Muda AF, Musa H., "Segmentation and Classification Analysis Techniques for Stroke based on Diffusion-Weighted Images", IAENG International Journal of Computer Science, vol. 44, no. 3, Aug 2017.

[17] Abdullah AR, Abidullah NA, Shamsudin NH, Ahmad NH, Jopri MH.,"Power Quality Signals Classification System Using Time-Frequency Distribution”, InApplied Mechanics and Materials, Trans Tech Publications, vol. 494, pp. 1889-1894, 2014.

[18] Abdullah AR, Abidullah NA, Shamsudin NH, Ahmad NH, Jopri MH.,"Performance Verification of Power Quality Signals Classification System", InApplied Mechanics and Materials, Trans Tech Publications, vol. 752, pp. 1158-1163, 2015.

[19] Jopri MH, Abdullah AR, Kassim NM, Manap M, Ngatiman NA, Yusoff MR.,"Localization of Multiple Harmonic Sources for Inverter Loads Utilizing Periodogram”, Journal of Telecommunication, Electronic and Computer Engineering (JTEC), vol. 8, no. 2, pp 87-91, May 2016.

[20] Habban, M.F., Manap, M., Abdullah, A.R., Jopri, M.H. and Sutikno, T., "An Evaluation of linear time frequency distribution Analysis for VSI switch faults identification", International Journal of Power Electronics and Drive Systems (IJPEDS), vol. 8, no.1, pp. 1-9.

[21] Jopri MH, Abdullah AR, Manap M, Habban MF, Sutikno T.,"An Accurate Classification Method of Harmonic Signals in Power Distribution System by Utilising S-Transform”, Telkomnika (, vol. 15, no. 1, Mar 2017. 


\section{BIOGRAPHIES OF AUTHORS}

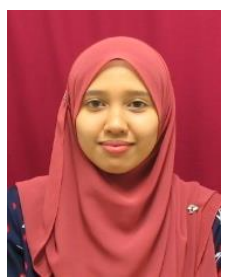

N. S. M. Noor received B. Eng of electronic and computer engineering from Universiti Teknikal Malaysia Melaka (UTeM) in 2016. She is currently pursuig M. Sc degree in Electronic Engineering at UTeM.Her main research interest is in the field of image processing and machine learning.

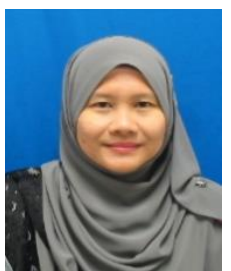

N. M. Saad is a senior lecturer at Department of Computer Engineering, Faculty of Electronics and Computer Engineering, Universiti Teknikal Malaysia Melaka (UTeM). She received her B. Eng. in Medical Electronics (2001), Master Eng. in Telecommunications (2004) and PhD in Digital Image Processing (2015) from Universiti Teknologi Malaysia (UTM). Her research area involves Digital Image and Signal Processing, Computer Vision and Medical Imaging. She is registered with Board of Engineer Malaysia (BEM), Malaysia Board of Technologist (MBOT), Institute for Electrical and Electronics Engineers (IEEE) Signal Processing Society (SPS), IEEE Engineering in Medicine and Biology Society (EMBS) and International Association of Engineers (IAENG).

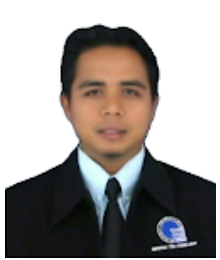

A.R. Abdullah received his B. Eng. in Electrical Engineering (2001), Master degree in Electrical Engineering (2004) and PhD degree in Power Engineering and Digital Signal Processing (2011) from Universiti Teknologi Malaysia (UTM). He is currently an Associate Professor in the Department of Power Electronics \& Drive, Faculty of Electrical Engineering, Universiti Teknikal Malaysia Melaka (UTeM) andHead of AdvancedDigital Signal Processing (ADSP) Lab. He is currently active in the Center of Excellence $(\mathrm{CoE})$ andlead for E-Beca for Universiti Teknikal Malaysia Melaka (UTeM). His field of specialization includes advanced digital signal processing for power quality, rehabilitation engineering, assistive technology and power electronics and drives.

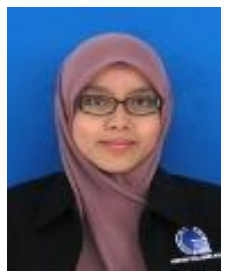

N. M. Ali received B. Eng (Hons.) (Mechatronic Engineering) from the Universiti Teknikal Malaysia Melakain 2009 and M. Sc (Mechatronic Engineering) from Universiti Islam Antarabangsa in 2014.She is currently pursuing PhD degree in Electrical Engineering at Multimedia University, Melaka. Her main research interest is in the field of image processing, intelligent system and machine learning. 